\title{
Faculty As Filmmakers: On The Cutting Edge Of Classroom Technologies
}

Susan G. Rozensher, (Email: SRozensher@iona.edu), Iona College

\begin{abstract}
Digital video equipment and studios newly available for faculty use can be incorporated into the technological repertoire of college faculty, enabling professors to customize and enhance the learning experience of their students. Today's students are particularly attuned to analyzing visual images and data, so the use of customized films in the classroom makes very good sense from a pedagogical standpoint. The present paper examines the lessons learned from the process of producing a documentary-style digital video that serves as the centerpiece of a thought-provoking learning module for the introductory course in marketing.
\end{abstract}

\section{OVERVIEW}

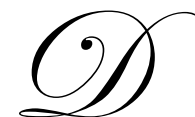

igital video equipment and studios newly available for faculty use can be incorporated into the technological repertoire of college faculty, enabling professors to customize and enhance the learning experience of their students. Today's students are particularly attuned to analyzing visual images and data, so the use of customized films in the classroom makes very good sense from a pedagogical standpoint. The present paper examines the lessons learned from the process of producing a documentary-style digital video that serves as the centerpiece of a thought-provoking learning module for the introductory course in marketing*. Faculty considering such an undertaking should be encouraged to learn that the author undertook this project without any prior experience in the production of a video.

\section{TOPIC SELECTION}

The selection of the topic for the video is the most important decision to be made by the prospective filmmaker. One key criterion for choosing a successful topic is its relevance to the students. The video that the author created focuses on the controversial growth of marketing activities inside high schools, including advertising to students through video news programs shown during class, advertising in school corridors, and the sale of certain products in schools, such as cola beverages. Ethical, political, psychological and social concerns are propelling this new "frontier" of business into a subject of serious debate. This particular topic serves as a useful illustration of the types of conflicts that arise when serious consideration is given to the "societal marketing" concept - a concept that is generally covered in introductory marketing classes. The topic was selected for the video because of its relevance to college students, who would be able to relate to the issue's complexity by drawing on many of their own experiences and opinions.

For the purposes of lively class discussion, it is helpful to choose topics that engender debate, with more than one tenable position for college students to espouse. Given the cultural climate on campus, it is worthwhile to consider whether students would feel comfortable supporting different positions - i.e. there should be more than one socially acceptable position for a college student to champion in order to generate a productive debate.

\footnotetext{
*Funding for the project was provided by an Iona College Faculty Instructional Technology Award.
} 


\section{INTERVIEWEE SELECTION}

Documentaries tend to rely heavily on experts in an attempt to present a range of well-informed opinions. A very useful technique for building curiosity about the conflicting viewpoints in a controversy centers on the careful selection of various stakeholders to be interviewed.

One of the most successful techniques that the author used for generating interest in the film's topic involved the interviewing of several students on the college campus where the author serves on the faculty. These interviews served to enhance students' identification with the material and their eagerness to debate varying points of view. Interviews of students from other schools were also included in the video to add more interest. The student interviews were incorporated into the video after interviews with several experts on the topic had been presented.

Eliciting the cooperation of experts can be quite challenging. Some experts may prove to be very concerned about the legal or political ramifications of such an interview, and others find it necessary to obtain permission from their employers (permission which may or may not be granted). In some cases it may be more fruitful to solicit founders and partners in companies; they are more likely to agree to an interview and can avoid bureaucratic obstacles. Employees of non-profit organizations may also prove to be more enthusiastic about participating in an interview.

\section{LEGAL CONCERNS}

When interviewees were solicited for taping, they were advised to consult a website where a description of the grant that the author received could be readily accessed. That website was located on the official website of the college where the author is a faculty member. The website described the nature of the video and its intended educational purposes while lending legitimacy to the effort.

It is worthwhile to request written consent from each interviewee to show as well as publish the taped material for educational purposes, in order to prevent the development of any possible legal concerns. Written consent should be requested before or at the time of the interview, when interviewees are most enthusiastic about the endeavor and willing to give permission. Such planning will also prevent the practical problem of locating interviewees later on; students in particular can be difficult to locate since their addresses and phone numbers tend to change frequently.

Permission must be obtained to use copyright-protected material, such as background music or visual images such as clips from other video material. Such material would be considered "republished" and would require permission to use. Permission must also be acknowledged in the video credits. Problems and costs associated with obtaining such permission may inhibit the use of copyright-protected material. For more information about copyright issues, consult the Copyright Clearance Center online.

\section{COMMUNICATION TECHNIQUES}

Perhaps the most important recommendation the author can make is to seek out the assistance of a faculty member in the communications department or film school. Such a seasoned professional will help the filmmaker avoid many basic mistakes in the areas of lighting and sound. As a result, the final product will have a more professional look - an attribute that students definitely value and expect (Seal and Przasnyski, 2003).

Interviewing presents many challenges and options. If no assistant is available, faculty may find themselves responsible for handling the video equipment while simultaneously conducting the interview. This was the case for most of the interviews that the author conducted, since many of them did not take place on the college campus.

There are several options available for conducting an interview in such circumstances, when only one video camera is available. One option, ultimately chosen by the author, was to set up a tripod with a stationary camera focused on the interviewee. Later the author arranged to be videotaped in the studio on campus (on a blank background), asking the same questions that the author had asked previously in the actual interview. During the editing 
phase, the author was able to piece the two tapes together to create an interview that generally seemed realistic. This did require a great deal of effort because of many technical issues concerning, for instance, the audio track.

Another worthwhile option is to videotape oneself (after setting up the camera on a tripod) right after completing the interview, asking the same questions asked during the interview. The advantage here is that the same setting and lighting can be used, to enhance the realistic appearance of the interview. It is also helpful to tape some "reaction shots" in which the interviewer is shown nodding, or silent and listening intently. These shots can later be integrated during the editing phase where necessary.

It is also possible to eliminate entirely the need for an interviewer in the video by simply asking interviewees to incorporate the questions into their responses. From time to time, this may require re-taping of a response to a question when the interviewee forgets to incorporate the question.

If an assistant is available, many other options can be utilized. Before or after the interview, both interviewer and interviewee can be taped simultaneously from a distance in a "cover shot" that is too far away to see what exactly they are saying. This shot, together with clips of the interviewer and others of the interview, give the impression that there are three cameras. As a result, the final videotape will have a more professional look.

\section{SCRIPTING}

The experience of the author led to the conclusion that a script is a very useful and probably necessary device for planning and narrating the video, and for preparing questions to be asked. Scripting helps to keep the segments coherent and provides a logical flow. It also helps to insure that the content is well focused. During the editing process, the script serves as a vehicle for organizing the numerous video clips into a story along the time line that makes sense to the viewer and that conveys information effectively. Although the author did not use a storyboard, such a tool can be very useful as well.

\section{EDITING}

The digital video editing software that the author used was Canopus StormEdit, which is similar to Adobe Premiere. Editing proved to be the most challenging and time-consuming aspect of the production of the video. The first step involved capturing the footage from the digital videotapes to create and name video clips. The next step involved reviewing the clips and trimming them, setting In and Out points, and organizing them along a time line. Eventually, transitions (such as fading to black) were inserted to smooth out the linking together of the individual clips. Finally, titles were designed and inserted. Special effects can be added as well. This entire process of editing required a great deal of attention to detail in order to create a final product with a reasonably professional appearance.

\section{TIME INVESTMENT}

There are many pedagogic benefits to be reaped from the production of a digital video, and students seem very enthusiastic about the use of customized videos for igniting their interest in and understanding of complex issues. However, the effort involved in producing a video requires a large investment of time and effort, from the planning and scripting stages, to the conducting of interviews, to the editing of the final product. Individual instructors will have to conduct their own cost/benefit analyses to decide whether the effort is worthwhile. Undoubtedly, future advances in the user-friendliness of editing software will serve to enhance the attractiveness of video production as a pedagogical tool.

\section{REFERENCES}

1. Seal, Kala C. and Zbigniew Przasnyski (2003), Using technology to support pedagogy in an OR/MS course, Interfaces. Jul/Aug, Vol. 33, Iss. 4, pp. 27. 
NOTES 